\title{
Effect of maternal smoking during pregnancy on childhood type 1 diabetes: a whole-of-population study
}

\author{
Mumtaz Begum ${ }^{1,2,3}$ (1) $\cdot$ Rhiannon M. Pilkington ${ }^{1,2}$ (I) $\cdot$ Catherine R. Chittleborough ${ }^{1,2}$ (D) $\cdot$ John W. Lynch ${ }^{1,2,4}$ (I) \\ Megan Penno ${ }^{2,5}$ (D) Lisa G. Smithers ${ }^{1,2}$ (1)
}

Received: 15 November 2019 / Accepted: 15 January 2020/Published online: 24 February 2020

(C) Springer-Verlag GmbH Germany, part of Springer Nature 2020

\begin{abstract}
Aims/hypothesis Evidence of an association between maternal smoking during pregnancy (prenatal smoking) and childhood type 1 diabetes is mixed. Previous studies have been small and potentially biased due to unmeasured confounding. The objectives of this study were to estimate the association between prenatal smoking and childhood type 1 diabetes, assess residual confounding with a negative control design and an E-value analysis, and summarise published effect estimates from a meta-analysis.

Methods This whole-of-population study (births from 1999 to 2013, participants aged $\leq 15$ years) used de-identified linked administrative data from the South Australian Early Childhood Data Project. Type 1 diabetes was diagnosed in 557 children (ICD, tenth edition, Australian Modification [ICD-10-AM] codes: E10, E101-E109) during hospitalisation (2001-2014). Families not given financial assistance for school fees was a negative control outcome. Adjusted Cox proportional HRs were calculated. Analyses were conducted on complete-case $(n=264,542$, type 1 diabetes $=442)$ and imputed $(n=286,058$, type 1 diabetes $=557$ ) data. A random-effects meta-analysis was used to summarise the effects of prenatal smoking on type 1 diabetes. Results Compared with non-smokers, children exposed to maternal smoking only in the first or second half of pregnancy had a $6 \%$ higher type 1 diabetes incidence (adjusted HR 1.06 [95\% CI 0.73, 1.55]). Type 1 diabetes incidence was 24\% lower (adjusted HR 0.76 [95\% CI $0.58,0.99]$ ) among children exposed to consistent prenatal smoking, and $16 \%$ lower for exposure to any maternal smoking in pregnancy (adjusted HR 0.84 [95\% CI 0.67, 1.08]), compared with the unexposed group. Meta-analytic estimates showed 28-29\% lower risk of type 1 diabetes among children exposed to prenatal smoking compared with those not exposed. The negative control outcome analysis indicated residual confounding in the prenatal smoking and type 1 diabetes association. E-value analysis indicated that unmeasured confounding associated with prenatal smoking and childhood type 1 diabetes, with a HR of 1.67, could negate the observed effect.

Conclusions/interpretation Our best estimate from the study is that maternal smoking in pregnancy was associated with $16 \%$ lower childhood type 1 diabetes incidence, and some of this effect was due to residual confounding.
\end{abstract}

Keywords Childhood $\cdot$ E-value $\cdot$ Maternal smoking $\cdot$ Negative control outcome $\cdot$ Population-based birth cohort $\cdot$ Pregnancy Type 1 diabetes

Electronic supplementary material The online version of this article (https://doi.org/10.1007/s00125-020-05111-w) contains peer-reviewed but unedited supplementary material, which is available to authorised users.

Lisa G. Smithers

lisa.smithers@adelaide.edu.au

John W. Lynch

john.lynch@adelaide.edu.au

1 School of Public Health, The University of Adelaide, Level 9, AHMS Building, North Terrace, Adelaide, SA 5005, Australia
2 Robinson Research Institute, The University of Adelaide, Adelaide, SA, Australia

3 Department of Food and Nutrition, College of Home Economics, University of Peshawar, Peshawar, Pakistan

4 Population Health Sciences, University of Bristol, Bristol, UK

5 Adelaide Medical School, The University of Adelaide, Adelaide, SA, Australia 


\section{Research in context}

\section{What is already known about this subject?}

- Evidence is mixed about maternal smoking in pregnancy and risk of childhood type 1 diabetes

- Most case-control and population-based cohort studies reported reduced type 1 diabetes risk for children exposed to maternal smoking in pregnancy

\section{What is the key question?}

- What is the effect of maternal smoking during pregnancy on risk of childhood type 1 diabetes and what is the potential for bias in this effect due to unmeasured confounding?

\section{What are the new findings?}

- Maternal smoking in pregnancy was associated with 16\% lower childhood type 1 diabetes incidence

- Our meta-analytic estimates demonstrated 28-29\% reduced type 1 diabetes risk for children exposed to maternal smoking in pregnancy

- The negative control analysis indicated that some of the observed effect of prenatal maternal smoking on childhood type 1 diabetes was due to residual confounding. The E-value indicated that unmeasured confounding associated with prenatal maternal smoking and childhood type 1 diabetes, with a HR of 1.67 , could negate the observed effect

\section{How might this impact on clinical practice in the foreseeable future?}

- Maternal smoking in pregnancy is not recommended but the mechanism leading to reduced type 1 diabetes should be investigated

\section{Introduction}

Onset of type 1 diabetes can occur at any age, but many children who develop this condition have detectible autoantibodies targeting beta cell antigens within the first year of life [1], suggesting that early exposures may have a role in the natural history of type 1 diabetes [2]. Increasing trends, country variation in global type 1 diabetes incidence $(0.01-60$ per 100,000 population per-year) and the type 1 diabetes discordance in monozygotic twins all suggest a role of non-genetic factors [3-5]. An environmental exposure implicated in type 1 diabetes pathogenesis is maternal smoking during pregnancy. Mechanisms that link prenatal smoking and childhood type 1 diabetes are not understood but may be associated with altered gene expression or immune function $[6,7]$.

Type 1 diabetes incidence has increased in many countries that are ranked in the top ten for type 1 diabetes incidence, including Finland, Sweden, the USA and UK [3, 8], while prenatal smoking rates have declined or become stable in these countries in the last decade [9]. In Australia, type 1 diabetes incidence among $0-14$ year olds has increased from 21.5 per 100,000 population in 2000 , to 24.7 per 100,000 population in 2015 [10], while prevalence of smoking during pregnancy reduced from $17.3 \%$ in 2006 [11] to $9.9 \%$ in 2016 [12]. Previous studies of prenatal smoking and childhood type 1 diabetes reported mixed findings, demonstrating increased [13], decreased [14-16] and null [17] type 1 diabetes risk. A
Swedish HLA-genotype-matched case-control study demonstrated an increased type 1 diabetes risk for children exposed to prenatal smoking [13], and a UK record-linkage study did not find any difference in the type 1 diabetes incidence between children exposed and unexposed to prenatal smoking [17]. Conversely, large population-based cohort studies have demonstrated 25-35\% lower type 1 diabetes risk for children exposed to prenatal smoking $[15,16]$. These mixed findings of an association between prenatal smoking and childhood type 1 diabetes could be due to differences in confounding adjustments. Many population-based studies have adjusted for confounders such as maternal age [13-16], maternal deprivation or socioeconomic position [14, 15], birth order or parity [13, 15, 16], maternal education, pre-pregnancy BMI and diabetes, and some have adjusted for mediators [13-15]. Some studies have not adjusted for father's age, pre-pregnancy diabetes or hypertension, ethnicity or socioeconomic indicators, and one study excluded children whose mothers had pre-existing diabetes [13-15]. Most studies on smoking during pregnancy and type 1 diabetes risk have small numbers of children exposed to smoking during pregnancy, ranging from 5 to 72 in population-based cohort studies $[15,16,18]$ and 29 to 258 in case-control studies [13, 14, 19-22]. Small sample sizes could be another reason for variable findings in previous studies; therefore, one way to obtain a more precise effect estimate of the prenatal smoking and type 1 diabetes association is to combine these estimates in a meta-analysis. 
The objectives of this study were: (1) to estimate the association between prenatal maternal smoking and childhood type 1 diabetes incidence with adjustment for a range of confounding factors defined a priori; (2) to measure the potential for bias due to unmeasured confounding using a negative control outcome analysis and E-value calculation; and (3) to perform a meta-analysis of published population-based cohort and case-control studies.

\section{Methods}

Study population and design We used data from the South Australian Early Childhood Data Project, which consists of routinely collected, de-identified, linked government administrative datasets [23]. Datasets were linked by an independent agency (SA NT Datalink; www.santdatalink.org.au, accessed 1 May 2019) [24]. Children were linked across datasets using a probabilistic algorithm that included demographic information, such as name, date of birth, sex and address. A $0.5 \%$ false linkage rate has been reported in Australia [24]. This study used inpatient hospitalisation data from July 2001 to June 2014, and perinatal and birth registration data from 1999 to 2013 (Fig. 1).

Type 1 diabetes Individuals with type 1 diabetes (aged $\leq 15$ years) were identified from inpatient hospitalisation data from all public hospitals in South Australia. Children with index type 1 diabetes hospitalisations were identified using ICD, tenth edition, Australian Modification (ICD-10-AM) codes (E10, ranging from E101 to E109), [25], including both principal and additional diagnoses. Diagnoses codes were assigned to each hospitalisation episode by trained hospital staff.

Maternal smoking during pregnancy Data on prenatal smoking was obtained from the South Australian Perinatal Statistics Collection [26] from 1999 to 2013. It is mandatory for perinatal statistics to be collected for every child born in South Australia. Data were collected by midwives or nurses using a standard data collection form. Perinatal data have been collected by the South Australian government since 1981 to track mother and child health indicators $[12,26]$. The data collection form has been validated against an audit of medical records [27].

Maternal smoking data were collected at the first antenatal visit ( $\leq 20$ weeks' gestation) and in the second half of pregnancy ( $\geq 20$ weeks' gestation) [26]. Most women (74.3\%) had their first antenatal visit before 14 weeks' gestation [26]. For the primary analysis, smoking was categorised into nonsmokers, smokers only in first or second half of pregnancy, and consistent smokers. For women who smoked only in the first or second half of pregnancy, no information is available on when they started or stopped smoking, therefore, the duration of smoking is unclear. Due to small numbers of offspring with type 1 diabetes among women who smoked, smoking in pregnancy was dichotomised into non-smokers or smokers (including women who smoked any time during pregnancy) for secondary analyses.

Confounding Confounders of the association between maternal smoking during pregnancy and type 1 diabetes risk were identified a priori based on literature and by using a directed
Fig. 1 Flow chart of the study population. Maternal BMI was measured from 2007 onwards. HP, hypertension; IRSAD, Index of Relative Socioeconomic Advantage and Disadvantage; SA, South Australian; T1D, type 1 diabetes

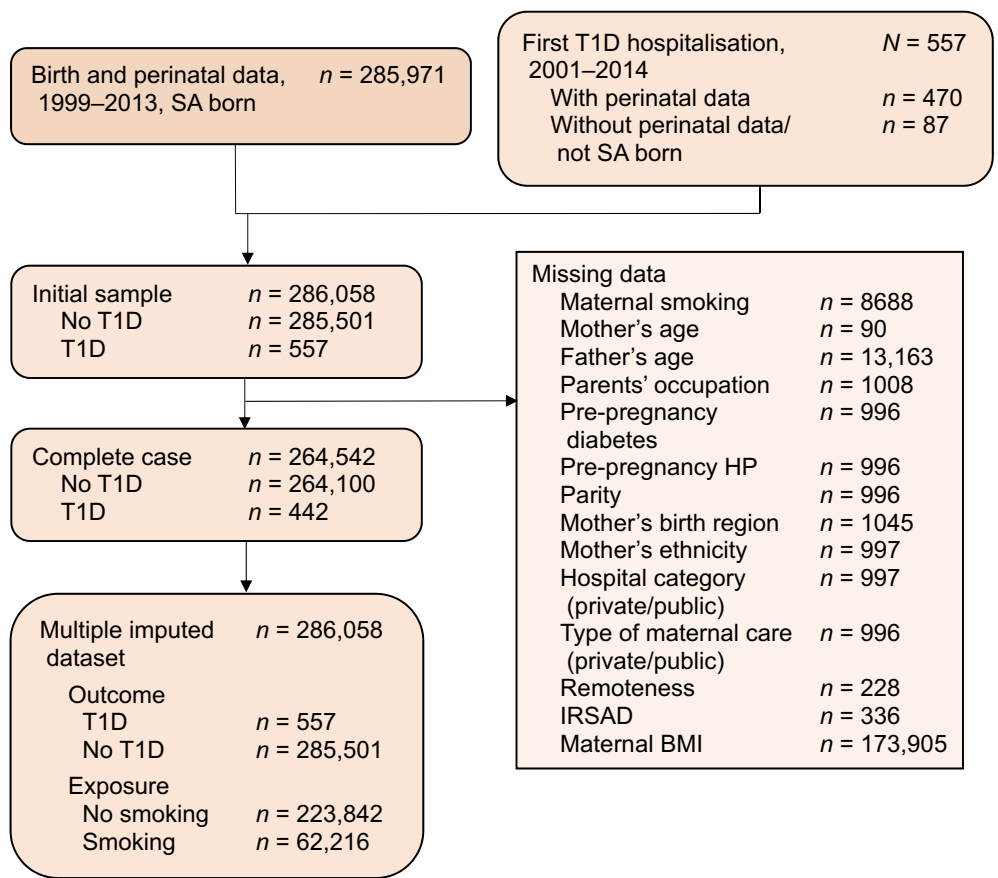


acyclic graph (electronic supplementary material [ESM] Fig. 1). Information on confounders was obtained from the South Australian Perinatal Statistics Collection [26]. Parental characteristics included mother's and father's age (continuous variables), and parental highest occupation in four categories: (1) managers, administrators and professionals; (2) paraprofessionals, tradespersons, clerks, salespersons and personal service; (3) plant and machine operators, drivers and labourers; and (4) students, pensioners, home duties and unemployed. Maternal and child characteristics were maternal birth region (Oceania, Europe, or Africa, Asia or America), mother's ethnicity (European descent, Aboriginal or Torres Strait Islander, or Asian and other), whether the mother was a private or public hospital patient, type of hospital where the child was born (private/public), maternal pre-pregnancy hypertension (yes/no), pre-pregnancy diabetes (yes/no), parity (first, second, third or $\geq$ fourth) and the child's year of birth. Area-level measures of socioeconomic conditions (Index of Relative Socioeconomic Advantage and Disadvantage [IRSAD]), and remoteness and accessibility (Australian Remoteness Index for Areas) were based on the mother's postcode at the time of birth. Maternal BMI at the first antenatal visit $\left(<25 \mathrm{~kg} / \mathrm{m}^{2}, 25\right.$ to $<30 \mathrm{~kg} / \mathrm{m}^{2}$ or $\left.\geq 30 \mathrm{~kg} / \mathrm{m}^{2}\right)$ was included as a confounder only in sensitivity analysis as maternal BMI data were not collected for births prior to 2007 .

Statistical analysis The crude and adjusted association between prenatal smoking and childhood type 1 diabetes for both primary and secondary analyses was estimated by Cox proportional hazard regression, to account for differences in observation time across successive birth years. These analyses were conducted on both complete-case (ESM Table 2; $n=$ 264,542, type 1 diabetes $=442)$ and imputed (Table 2; $n=$ 286,058 , type 1 diabetes $=557$ ) data. Schoenfeld residual tests demonstrated non-violation of the proportional hazard assumption. Children were followed from birth until the diagnosis of type 1 diabetes or the end of follow-up (June 2014). The observation time ranged from 1 year (for births in 2013) to 15 years (for births in 1999), with a mean follow-up of 8 years.

A negative control outcome analysis was used to investigate whether any association between maternal smoking in pregnancy and type 1 diabetes could be due to unmeasured confounding [28]. Cox proportional hazard regression analysis estimated the association between maternal smoking during pregnancy and the family not having a school card for financial assistance with school fees. An assumption about negative control outcomes is that the measured and unmeasured confounding pattern for the association between maternal smoking and type 1 diabetes is the same as for the maternal smoking and no school card association (called 'U-comparable') [28]. There is no plausible reason for prenatal maternal smoking to directly cause a child to get a school card. If there is any association between maternal smoking in pregnancy and child not having a school card, it must be through confounders (e.g. sociodemographic characteristics), additional backdoor paths or unmeasured confounding (ESM Fig. 2). School card data was sourced from the school enrolment census and was provide by the Department for Education, South Australia. Data were only available for children who had started school (complete-case analysis: $n=$ 277,370 [no school card, $n=84,531$ ]; imputed data: $n=$ 184,663 [no school card, $n=149,670$ ]).

The E-value was calculated to measure the potential for bias due to unmeasured confounding in the prenatal smoking and type 1 diabetes association. The E-value quantifies the minimum strength of an association that an unmeasured confounder would need to have with the exposure and outcome to negate the observed association between prenatal smoking and type 1 diabetes, given the measured confounders [29]. The E-value for the CI quantifies the strength of an association that unmeasured confounding would need to have with prenatal smoking and childhood type 1 diabetes, above and beyond the measured confounders, to change the CI to include the null [29].

Five sensitivity analyses were performed to see if the association between prenatal smoking and childhood type 1 diabetes was similar to the main findings. In the first sensitivity analysis, we examined whether adjusting for pre-pregnancy BMI (in addition to all other confounders) influenced the association between prenatal smoking and type 1 diabetes. Maternal BMI was only collected from 2007 onwards and, therefore, could not be included in the main analyses. The second sensitivity analysis was restricted to births from July 2001 to December 2013, as hospital data was only available from July 2001 and any diagnosis of type 1 diabetes from 1999 to mid-2001 may have been misclassified. As perinatal data was only collected for South Australian-born children, the third sensitivity analysis was restricted to children born in South Australia, for whom more complete data were available. The fourth sensitivity analysis was restricted to singleton births to make our study comparable with previous studies that were conducted only on singleton births $[15,16]$. Finally, all the above were combined and the analysis was restricted to singleton children born in South Australia from July 2001 to December 2013. All the sensitivity analyses were adjusted for maternal BMI along with all other confounders.

The amount of missing information on the exposure and confounders in Table 1 ranged from $0.03 \%$ to $0.37 \%$, except prenatal maternal smoking (3.0\%), father's age $(4.6 \%)$ and maternal BMI (66.6\%; not included in primary analysis). Multiple imputation by chained equations was conducted to maintain the association between prenatal smoking and childhood type 1 diabetes and to account for potential bias if the association differed between children with and without complete data [30]. The outcome variable (type 1 diabetes) 
Table 1 Characteristics of children by maternal smoking during pregnancy $(N=286,058)$

\begin{tabular}{|c|c|c|c|c|}
\hline \multirow[t]{2}{*}{ Characteristic } & \multicolumn{2}{|c|}{$\begin{array}{l}\text { Imputed analysis } \\
N=286,058^{\mathrm{a}}\end{array}$} & \multicolumn{2}{|c|}{$\begin{array}{l}\text { Complete-case analysis } \\
N=264,542\end{array}$} \\
\hline & $\begin{array}{l}\text { No smoking } \\
n=223,842\end{array}$ & $\begin{array}{l}\text { Smoking } \\
n=62,216\end{array}$ & $\begin{array}{l}\text { No smoking } \\
n=212,414\end{array}$ & $\begin{array}{l}\text { Smoking } \\
n=52,128\end{array}$ \\
\hline \multicolumn{5}{|l|}{ Type 1 diabetes } \\
\hline No & $223,403(99.8)$ & $62,098(99.8)$ & $212,064(99.8)$ & $52,036(99.8)$ \\
\hline Yes & $439(0.2)$ & $118(0.2)$ & $350(0.2)$ & $92(0.2)$ \\
\hline Mother's age (years) & $30.1 \pm 5.4$ & $27.4 \pm 6.1$ & $30.1 \pm 5.3$ & $27.6 \pm 6.0$ \\
\hline Father's age (years) & $33.2 \pm 6.4$ & $31.0 \pm 7.0$ & $33.3 \pm 6.3$ & $31.1 \pm 6.9$ \\
\hline \multicolumn{5}{|l|}{ Pre-pregnancy diabetes } \\
\hline No & $222,591(99.4)$ & $61,759(99.3)$ & $211,283(99.5)$ & $51,774(99.3)$ \\
\hline Yes & $1251(0.6)$ & $457(0.7)$ & $1131(0.5)$ & $354(0.7)$ \\
\hline \multicolumn{5}{|l|}{ Pre-pregnancy HT } \\
\hline No & $221,030(98.7)$ & $61,475(98.8)$ & $209,763(98.8)$ & $51,524(98.8)$ \\
\hline Yes & $2812(1.3)$ & $741(1.2)$ & $2651(1.2)$ & $604(1.2)$ \\
\hline \multicolumn{5}{|l|}{ Hospital category (for childbirth) } \\
\hline Private & $67,304(30.1)$ & $5191(8.3)$ & $65,446(30.8)$ & $4830(9.3)$ \\
\hline Public & $156,538(69.9)$ & $57,025(91.7)$ & $146,968(69.2)$ & $47,298(90.7)$ \\
\hline \multicolumn{5}{|l|}{ Type of patient (mother, healthcare) } \\
\hline Private & $81,670(36.5)$ & $6704(10.8)$ & $78,018(36.7)$ & $6014(11.5)$ \\
\hline Public & $142,172(63.5)$ & $55,512(89.2)$ & $134,396(63.3)$ & $46,114(88.5)$ \\
\hline \multicolumn{5}{|l|}{ Parity } \\
\hline 1st child & $95,457(42.6)$ & $24,419(39.2)$ & $90,323(42.5)$ & $20,622(39.6)$ \\
\hline 2nd child & $81,299(36.3)$ & $18,754(30.1)$ & $77,871(36.7)$ & $16,098(30.9)$ \\
\hline 3rd child & $32,074(14.3)$ & $10,392(16.7)$ & $30,440(14.3)$ & $8696(16.7)$ \\
\hline$\geq 4$ th child & $15,011(6.7)$ & $8650(13.9)$ & $13,780(6.5)$ & $6712(12.9)$ \\
\hline \multicolumn{5}{|l|}{ Parents' highest occupation } \\
\hline Manager/administrator/professional & $103,424(46.2)$ & $12,088(19.4)$ & $99,549(46.9)$ & $10,994(21.1)$ \\
\hline $\begin{array}{l}\text { Para-professional/ tradesperson/clerk/ } \\
\text { salesperson/personal service }\end{array}$ & $83,595(37.3)$ & $24,295(39.0)$ & $80,009(37.7)$ & $21,578(41.4)$ \\
\hline Plant and machine operators/drivers/labourers & $20,554(9.2)$ & $12,203(19.6)$ & $19,459(9.2)$ & $10,598(20.3)$ \\
\hline Students/pensioners/home duties/unemployed & $16,269(7.3)$ & $13,630(21.9)$ & $13,397(6.3)$ & $8958(17.2)$ \\
\hline \multicolumn{5}{|l|}{ Mother's birth region } \\
\hline Oceania & $180,678(80.7)$ & $58,150(93.5)$ & $171,221(80.6)$ & $48,533(93.1)$ \\
\hline Europe & $17,029(7.6)$ & $2869(4.6)$ & $16,268(7.7)$ & $2528(4.8)$ \\
\hline Africa/Asia/America & $26,135(11.7)$ & $1197(1.9)$ & $24,925(11.7)$ & $1067(2.0)$ \\
\hline \multicolumn{5}{|l|}{ Mother's ethnicity } \\
\hline White & $192,995(86.2)$ & $55,577(89.3)$ & $183,646(86.5)$ & $47,587(91.3)$ \\
\hline Aboriginal/Torres Strait Islander & $3283(1.5)$ & $5083(8.2)$ & $2543(1.2)$ & $3191(6.1)$ \\
\hline Asian/other & $27,564(12.3)$ & $1555(2.5)$ & $26,225(12.3)$ & $1350(2.6)$ \\
\hline \multicolumn{5}{|l|}{ Remoteness } \\
\hline Major cities & $166,731(74.5)$ & $40,835(65.6)$ & $158,572(74.7)$ & $34,628(66.4)$ \\
\hline Inner regional & $21,161(9.5)$ & $6147(9.9)$ & $20,054(9.4)$ & $5165(9.9)$ \\
\hline Outer regional/remote & $35,950(16.1)$ & $15,233(24.5)$ & $33,788(15.9)$ & $12,335(23.7)$ \\
\hline \multicolumn{5}{|l|}{ IRSAD $^{\mathrm{b}}$} \\
\hline 1st quintile & $54,062(24.2)$ & $25,441(40.9)$ & $50,598(23.8)$ & $20,661(39.6)$ \\
\hline 2nd quintile & $48,338(21.6)$ & $15,359(24.7)$ & $45,861(21.6)$ & $12,888(24.7)$ \\
\hline 3rd quintile & $38,442(17.2)$ & $9111(14.6)$ & $36,669(17.3)$ & $7827(15.0)$ \\
\hline 4th quintile & $47,752(21.3)$ & $8152(13.1)$ & $45,731(21.5)$ & 7154 (13.7) \\
\hline 5 th quintile & $35,247(15.7)$ & $4153(6.7)$ & $33,555(15.8)$ & $3598(6.9)$ \\
\hline
\end{tabular}


Table 1 (continued)

\begin{tabular}{|c|c|c|c|c|}
\hline \multirow[t]{2}{*}{ Characteristic } & \multicolumn{2}{|c|}{$\begin{array}{l}\text { Imputed analysis } \\
N=286,058^{\mathrm{a}}\end{array}$} & \multicolumn{2}{|c|}{$\begin{array}{l}\text { Complete-case analysis } \\
N=264,542\end{array}$} \\
\hline & $\begin{array}{l}\text { No smoking } \\
n=223,842\end{array}$ & $\begin{array}{l}\text { Smoking } \\
n=62,216\end{array}$ & $\begin{array}{l}\text { No smoking } \\
n=212,414\end{array}$ & $\begin{array}{l}\text { Smoking } \\
n=52,128\end{array}$ \\
\hline \multicolumn{5}{|l|}{ Maternal BMI ${ }^{\mathrm{c}}$} \\
\hline$<25 \mathrm{~kg} / \mathrm{m}^{2}$ (underweight/normal) & $114,211(51)$ & $29,006(46.6)$ & $38,046(50.4)$ & $6715(44.5)$ \\
\hline 25 to $<30 \mathrm{~kg} / \mathrm{m}^{2}$ (overweight) & $59,981(26.8)$ & $17,167(27.6)$ & $20,500(27.1)$ & $4044(26.8)$ \\
\hline$\geq 30 \mathrm{~kg} / \mathrm{m}^{2}$ (obese/severely obese) & $49,649(22.2)$ & $16,042(25.8)$ & $17,005(22.5)$ & $4327(28.7)$ \\
\hline
\end{tabular}

Data are presented as mean $\pm \mathrm{SD}$ or $n(\%)$

${ }^{a}$ For the imputed analysis, the $n$ values of the subgroups of the following do not equate to the total due to rounding: parity (both groups), mother's ethnicity (smoking), remoteness (smoking), IRSAD (no smoking), maternal BMI (both groups)

${ }^{\mathrm{b}}$ For IRSAD, first quintile is most disadvantaged, fifth quintile is most advantaged

${ }^{\mathrm{c}}$ Missing data: complete-case analysis/no smoking, $n=136,863$; complete-case analysis/smoking, $n=37,042$

HT, hypertension; IRSAD, Index of Relative Socioeconomic Advantage and Disadvantage

was not imputed. The outcome variable, all variables in Table 1 and the sensitivity analyses, and the Nelson-Aalen estimator of the cumulative hazard were included in the imputation models [31]. Summary statistics (Table 1) and estimates in Tables 2 and 3 were derived by combining the 20 imputed datasets, using Rubin's rules [32]. Analysis from completecase data are presented in ESM Table 2 and ESM Table 3.

Meta-analysis Even the largest studies investigating the effect of prenatal maternal smoking on type 1 diabetes only included relatively small numbers of children with type 1 diabetes who were born to mothers who smoked during pregnancy $(n=42-$ 72 in population-based cohort studies) $[15,16]$. Therefore, estimates from individual studies are imprecise. To compute a more precise estimate, a meta-analysis of the current results with previous studies that reported an association between prenatal smoking and childhood type 1 diabetes was conducted in January 2019. PubMed (www.ncbi.nlm.nih.gov), Web of Science (apps.webofknowledge.com), and Embase (www. embase.com) databases were systematically searched (accessed 8 December 2018) for studies on type 1 diabetes, maternal smoking during pregnancy and related terms, without limiting year of publication. Population-based studies written in English that reported maternal smoking during pregnancy as the exposure and overt or clinical type 1 diabetes in childhood ( $<19$ years $)$ as an outcome were included. Studies that reported beta cell autoimmunity (preclinical type 1 diabetes) as an outcome were excluded. Only populationbased studies with some attempt to adjust for confounding were included in the analysis.

Separate random-effects models were performed for the meta-analyses of population-based cohort and case-control studies. The meta-analysis of population-based studies pooled the HRs, and the meta-analysis of case-control studies pooled the odds ratios. All population-based-cohort studies reported HRs and all the case-control studies reported odds ratios. We
Table 2 Maternal smoking during pregnancy and risk of childhood type 1 diabetes

\begin{tabular}{lrll}
\hline Variable & T1D, $n$ & Unadjusted HR (95\% CI) & Adjusted HR (95\% CI) \\
\hline Maternal smoking in pregnancy & & & \\
$\quad$ Non-smoking & 439 & Ref. & Ref. \\
Smoked only in 1st/2nd half of pregnancy & 38 & $1.06(0.73,1.54)$ & $1.06(0.73,1.55)$ \\
Consistent smoking & 80 & $0.72(0.56,0.93)$ & $0.76(0.58,0.99)$ \\
Maternal smoking in pregnancy & & & Ref. \\
Non-smoking & 439 & Ref. & $0.84(0.67,1.08)$ \\
Smoking (any smoking in pregnancy) & 118 & $0.80(0.64,1.01)$ & \\
\hline
\end{tabular}

Analysis conducted on imputed data; $N=286,058$ (type 1 diabetes, $n=557$ )

a Adjusted for parents' age, parents' occupation, mother's birth region, maternal ethnicity, remoteness, IRSAD (Index of Relative Socioeconomic Advantage and Disadvantage), type of patient (mother), hospital category, parity, pre-pregnancy hypertension, pre-pregnancy diabetes and child's year of birth

${ }^{\mathrm{b}}$ Combination of consistent smoking and smoking only in first or second half of pregnancy

Ref., reference 
Table 3 Negative control outcome analysis: association between maternal smoking during pregnancy and child not having a school card

\begin{tabular}{|c|c|c|c|}
\hline Variable & $\begin{array}{l}\text { No school } \\
\text { card, } n\end{array}$ & $\begin{array}{l}\text { Unadjusted } \\
\text { HR (95\% CI) }\end{array}$ & Adjusted HR $(95 \% \mathrm{CI})^{\mathrm{a}}$ \\
\hline \multicolumn{4}{|l|}{ Maternal smoking in pregnancy } \\
\hline Non-smoking & 119,843 & Ref. & Ref. \\
\hline Smoked only in 1 st or 2 nd half of pregnancy & 8619 & $0.90(0.88,0.93)$ & $0.89(0.87,0.92)$ \\
\hline Consistent smoking & 21,208 & $0.80(0.78,0.81)$ & $0.85(0.83,0.87)$ \\
\hline \multicolumn{4}{|l|}{ Maternal smoking in pregnancy } \\
\hline Non-smoking & 119,843 & Ref. & Ref. \\
\hline Smoking (any smoking in pregnancy) ${ }^{\mathrm{b}}$ & 29,827 & $0.82(0.81,0.84)$ & $0.86(0.85,0.88)$ \\
\hline \multicolumn{4}{|l|}{$N=184,663($ no school card, $n=149,670)$} \\
\hline \multicolumn{4}{|c|}{$\begin{array}{l}\text { a'Adjusted for parents' age, parents' occupation, mother's birth region, maternal ethnicity, remoteness, IRSAD } \\
\text { (Index of Relative Socioeconomic Advantage and Disadvantage), type of patient (mother), hospital category, } \\
\text { parity, pre-pregnancy hypertension and pre-pregnancy diabetes }\end{array}$} \\
\hline \multicolumn{4}{|c|}{${ }^{\mathrm{b}}$ Combination of consistent smoking and smoking only in first or second half of pregnancy } \\
\hline
\end{tabular}

used a random-effects model as we did not assume homogeneity of effects among studies.

Analyses were conducted in Stata SE version 15.0 (StataCorp, College Station, TX, USA).

Ethical approval Ethical approval was granted by the Human Research Ethics Committees of the South Australian Department of Health (HREC/13/SAH/106) and the Aboriginal Health Council of South Australia (04-13-538).

\section{Results}

A total of 286,058 children (aged $\leq 15$ years) born from 1999 to 2013, contributed to 2,200,252 person-years of data. During follow-up 557 children were diagnosed with type 1 diabetes: an incidence of 25.3 per 100,000 person-years. Among 286,058 children, 62,216 were born to mothers who smoked during pregnancy.

Of the 557 children diagnosed with type 1 diabetes from 2001 to 2014,118 were exposed to maternal smoking during pregnancy, with 80 exposed to consistent smoking in both the first and second half of pregnancy. The crude type 1 diabetes incidence was 26.3 per 100,000 person-years for children not exposed, and 22.2 per 100,000 person-years for children exposed to maternal smoking.

The numbers in Table 1 show that, overall, socioeconomically disadvantaged women, who were from low income occupations, living in most disadvantaged areas, younger at the child's birth and delivered in public hospitals had higher prevalence of smoking during pregnancy. The distributions of these characteristics were similar in both the complete-case and imputed analyses (Table 1). There were numerically more socioeconomically disadvantaged women in the group that consistently smoked throughout pregnancy (ESM Table 1).
Primary and secondary analyses In the primary analysis (Table 2), following adjustment for confounding, type 1 diabetes incidence was $6 \%$ higher for children whose mothers smoked only in first or second half of pregnancy (HR 1.06 [95\% CI $0.73,1.55]$ ), and $24 \%$ lower for children exposed to consistent prenatal maternal smoking (HR 0.76 [95\% CI 0.58, 0.99]), compared with unexposed children. For smoking in the first or second half of pregnancy, CIs were wide and ranged from $27 \%$ reduced to $55 \%$ increased type 1 diabetes incidence. For consistent smoking, CIs ranged from $42 \%$ reduced to almost no difference in type 1 diabetes incidence between children exposed and unexposed to maternal smoking.

In secondary adjusted analysis, when the exposure included any smoking (first or second half of pregnancy and consistent smoking), childhood type 1 diabetes incidence was $16 \%$ lower for children exposed to maternal smoking in pregnancy (HR 0.84 [95\% CI 0.67, 1.08]) compared with those unexposed. Again, the confidence intervals were wide. Completecase analyses showed similar associations (ESM Table 2).

Potential for unmeasured confounding The negative control outcome analysis (Table 3 ) demonstrated $15 \%$ reduced incidence of not having a school card (HR 0.85 [95\% CI 0.83, $0.87]$ ) for children exposed to consistent prenatal maternal smoking, and $14 \%$ reduced incidence of not having a school card (HR 0.86 [95\% CI 0.85, 0.88]) related to exposure to any maternal smoking in pregnancy after adjustment for confounding. Complete-case analysis showed a similar pattern (ESM Table 3).

The E-value for the observed point-estimate (HR 0.84 [95\% CI $0.67,1.08]$ ) of prenatal smoking and childhood type 1 diabetes was 1.67. The observed $16 \%$ reduced incidence of type 1 diabetes for children exposed to prenatal smoking could be explained away by unmeasured confounding that was associated with prenatal smoking and childhood type 1 
diabetes by an HR of 1.67 each, above and beyond the measured confounders. The observed $95 \% \mathrm{CI}$ already included a null value $(95 \%$ CI $0.67,1.08)$, therefore, the E-value for the $\mathrm{CI}$ was 1 , suggesting that no unmeasured confounding would be needed to change the CI to include the null. For example in our study, type 1 diabetes in the father is a potential unmeasured confounder and a strong predictor of type 1 diabetes in the offspring (paternal type 1 diabetes vs no paternal type 1 diabetes, OR 9.19 [95\% CI 3.8, 22.0]) [21], which could indirectly have an impact on maternal smoking through the father's education and socioeconomic position [33]. As the E-value indicated the 'unmeasured confounder and outcome association' and the 'unmeasured confounder and exposure association', each would need to be equal to a HR of 1.67 to negate the observed effect. Although type 1 diabetes in the father is a strong predictor of type 1 diabetes in the offspring, we do not know the strength of the association between type 1 diabetes in the father and maternal smoking in pregnancy. In addition, the low prevalence of type 1 diabetes in the father $(<1 \%)[34]$ also reduces the potential to confound the maternal smoking and childhood type 1 diabetes association. Therefore, type 1 diabetes in the father may not be a strong enough unmeasured confounder to negate the observed effect of maternal smoking in pregnancy on childhood type 1 diabetes in this study.

Sensitivity analyses The sensitivity analyses (ESM Table 4) were consistent with the main findings.

Meta-analysis In addition to the current study, there were four previous population-based studies describing an association between prenatal smoking and type 1 diabetes available for the meta-analysis (Fig. 2). The meta-analysis showed $28 \%$ lower type 1 diabetes incidence for children exposed to prenatal smoking (HR 0.72 [95\% CI 0.62, 0.82]) compared with unexposed children. Similarly, the meta-analysis of six casecontrol studies (ESM Fig. 3) demonstrated 29\% reduced type 1 diabetes risk for children whose mothers smoked during pregnancy (OR 0.71 [95\% CI $0.55,0.86]$ ) compared with those children whose mothers had not smoked during pregnancy.

\section{Discussion}

In this large whole-of-population study, type 1 diabetes incidence was lower for children exposed to maternal smoking in pregnancy compared with children unexposed, after adjusting for a wide range of confounders. Similar to studies in this area, small numbers of type 1 diabetes cases among children exposed to prenatal smoking has impacted on the precision of the effect estimates. The CIs around the adjusted effect estimates in our primary and secondary analyses were wide but, on balance, provided some evidence to suggest a lower incidence of type 1 diabetes (consistent smokers, HR 0.76 [95\% CI 0.58, 0.99]). The crude absolute risk reduction was small, with four fewer type 1 diabetes cases per 100,000 person-years among children exposed to maternal smoking in pregnancy vs unexposed children. The $6 \%$ increased type 1 diabetes incidence for children whose mothers smoked only in the first or second half of pregnancy is difficult to interpret, again because of the very wide confidence intervals $(95 \% \mathrm{CI}$ $0.73,1.55$ ) and the small number of type 1 diabetes cases associated with mothers who smoked in the first or second half of pregnancy $(n=38)$. In addition, $69 \%$ of the 38 women who smoked only in the first or second half of pregnancy and

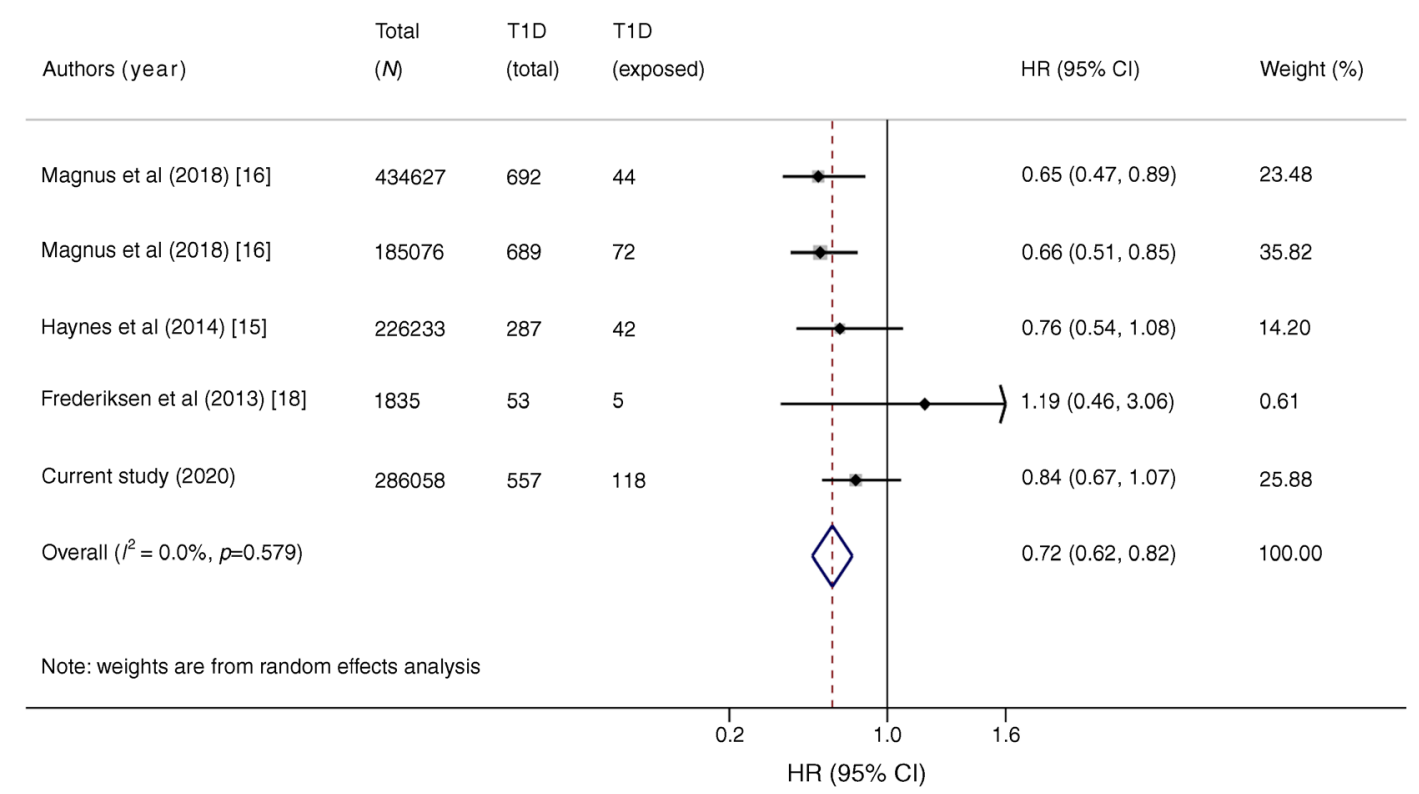

Fig. 2 Meta-analysis of maternal smoking during pregnancy and risk of type 1 diabetes (T1D) in population-based studies 
had a child with type 1 diabetes reported quitting before or at their first antenatal visit. This suggests that the exposure to smoking during pregnancy among this group was mostly limited to the first trimester. After triangulating across the main results and the meta-analysis, and despite the negative control outcome analysis indicating the likelihood of a small amount of unmeasured confounding, the evidence suggests a lower type 1 diabetes incidence for children exposed to maternal smoking during pregnancy as compared with unexposed children. However, because of the unmeasured confounding, the effect of smoking on type 1 diabetes is likely to be smaller than the point estimates suggest. As indicated by the E-value, unmeasured confounding associated with prenatal smoking and childhood type 1 diabetes, with an HR of 1.67, could negate the observed effect.

Smoking will never be recommended as an intervention for type 1 diabetes due to the significant harm it causes to both the mother and the fetus. The consequences of prenatal smoking have been well researched and include increased risk of miscarriage, preterm delivery, low birthweight, childhood obesity, respiratory problems, neurodevelopment and behavioural consequences $[35,36]$. It is not known which component of tobacco, nicotine or other combustible chemicals may be an active factor associated with reduced type 1 diabetes risk. Our findings suggest the mechanism of the effect of maternal smoking during pregnancy on type 1 diabetes (e.g. immune suppression by nicotine exposure, or alterations in gene expressions) needs further investigation.

The prevalence of smoking in the first half of pregnancy (23.8\% in $1999,11.2 \%$ in 2013) in our study is similar to reports of smoking in early pregnancy from Australia in 2013 (11.3\%) [37] and Scandinavia in 2009 (Denmark, 12.5\%; Norway, 16.5\%; Finland, 15\%) [38]. Of the women who reported smoking and gave birth from 1999 to 2013, 25\% quit at or before their first antenatal visit or only smoked in the first half of pregnancy, consistent with Australian national reports [12]. Consistent smoking in both the first and second half of pregnancy in our study (20.7\% in $1999,9.7 \%$ in 2013) is similar to Scandinavian estimates $(\sim 10 \%$ in Finland and Denmark in 2009) [38].

Our findings of a lower type 1 diabetes incidence following exposure to prenatal smoking were similar to findings of previous case-control and population-based cohort studies. However, the effect sizes in our study (16-24\%) were smaller than previously reported estimates (25-35\%) [14-16, 21, 22]. In our study, adjusting for a range of confounders did not considerably attenuate the effect estimate of maternal smoking on childhood type 1 diabetes. The number of children with type 1 diabetes exposed to prenatal smoking is small across previous studies, ranging from 42 to 72 in other population-based studies from Australia and Norway $[15,16]$. This is similar to our study, with 118 out of 557 children with type 1 diabetes exposed to maternal smoking in pregnancy. A meta-analysis was undertaken in our study to address the small samples and indicated that there was a $28 \%$ reduced risk of type 1 diabetes using data from population-based studies $[15,16,18]$ and a $29 \%$ reduced risk using data from casecontrol studies [13, 14, 19-22]. These results involve six countries (Norway, Denmark, Sweden, UK, USA and Australia) and 9872 children with type 1 diabetes, of which $16 \%$ were exposed to prenatal smoking. Our meta-analytic estimates are consistent with a recent meta-analysis [39]. However, our results are inconsistent with the HLA-matched case-control study that reported increased type 1 diabetes risk for children exposed to prenatal smoking [13]. Whilst the contribution of the HLA system may explain up to $60 \%$ of genetic risk of type 1 diabetes, it is plausible that other genetic risk variants contributing to susceptibility [40] may be more or less prevalent in type 1 diabetes cases compared with controls in the HLA-matched study.

Many studies, such as the Australian-based Environmental Determinants of Islet Autoimmunity (ENDIA) study [41] have been established in at-risk populations to elucidate mechanisms that lead to type 1 diabetes, and current thinking is that there are likely to be multiple mechanisms that link prenatal smoking with type 1 diabetes [42]. Epigenetic modifications induced by prenatal smoking, such as DNA methylation, may lead to changes in gene expression. Differences in DNA methylation have been reported among children exposed and unexposed to prenatal smoking [6], and among children with type 1 diabetes and their type 1 diabetes-discordant twins [43]. In addition, nicotine, a known immune suppressant reported to effect both the innate and adaptive immune responses, can pass through the placenta to fetal circulation [7]. The suppressive impact of smoking on immunity increases the risk of many chronic diseases, but there is some suggestion it could be protective for an autoimmune disease like type 1 diabetes. Smoking during pregnancy has been associated with reduced risks of other diseases with immune elements, such as preeclampsia, Parkinson's disease, ulcerative colitis and sarcoidosis [44-47]. The presence of autoantibodies in pre-eclampsia suggests that it may be a pregnancy-induced autoimmune disease [48]. High risk HLA genotypes for type 1 diabetes are also reported to be involved in ulcerative colitis [49], suggesting there may be a similar underlying mechanism of smoking in modifying the risk of both of these diseases.

This study included children attending all public hospitals in South Australia; therefore, children with type 1 diabetes who never attended any public hospital might have been missed. However, we believe that case ascertainment is high in this study because both primary and secondary diagnoses codes were used and, thus, even children who are hospitalised for other reasons were included if type 1 diabetes was noted as their secondary diagnosis. In addition, the South Australia paediatric hospital, with a specialised endocrinology unit and where children are admitted for stabilisation after diabetes diagnosis, was included in this study. In a similar setting in Western Australia, $99.8 \%$ of type 1 diabetes cases on the state 
diabetes register were ascertained in public hospital data [50]. Although social desirability bias in collecting maternal smoking is a common issue in observational studies, a Swedish validation study demonstrated that of the women who reported no smoking in pregnancy, $95 \%$ were classified as non-smokers based on serum cotinine concentration [51]. In our study, there could be some measurement error in maternal smoking during pregnancy. However, we do not have information about the extent of mismeasurement in the exposure to smoking as the data are not available. Furthermore, the capture of perinatal data was via a form validated against medical records. There may be misclassification of mother's tobacco exposure because we do not have data on father's smoking, which can affect the fetus through maternal passive smoking. Another limitation is the lack of information on dose or number of cigarettes smoked daily in pregnancy. We used the E-value and negative control outcome to indicate the potential for bias due to unmeasured confounding. The Evalue is dependent on the validity of the effect estimate and could be biased if there were selection and measurement biases. We have tried to improve the internal validity by adjusting for a range of potential confounders, using routinely collected data (reduced risk of recall bias) and conducting multiple imputation to account for bias due to loss of information. In addition, the negative control outcome analysis is assumed to have the exact same set of measured and unmeasured confounders (perfectly U-comparable) as the maternal smoking and childhood type 1 diabetes association. It is rare to have a perfectly U-comparable negative control outcome and it is most likely to only be approximately U-comparable [28]. However, because of non-perfect U-comparability, the negative control outcomes suggests that the association between smoking and type 1 diabetes is not entirely due to residual confounding.

Conclusion Our best estimate from this study is that there is a $16 \%$ reduced incidence of type 1 diabetes for children exposed to any maternal smoking in pregnancy as compared to unexposed children, but some of this effect is likely due to residual confounding. With the current analyses we cannot rule out an effect of maternal smoking during pregnancy on childhood type 1 diabetes. However, the absolute reduction in type 1 diabetes cases among children exposed to smoking was small (4 cases per 100,000 person-years). This study, along with the results of similar population-based studies, suggests that the mechanism leading to the association between maternal smoking and type 1 diabetes needs to be investigated.

Acknowledgements The authors thank the data custodians for contributing de-identified data. The data custodians include: South Australian Government office of Births, Deaths and Marriages; Department of Health and Wellbeing, SA Health; and Department for Education, South Australia. We thank SA NT Datalink (www.santdatalink.org.au) for the data linkage. The authors also thank M. Mittinty (School of Public
Health, The University of Adelaide, SA, Australia) for his guidance with statistical analyses. The findings and views reported are those of the authors and should not be attributed to any particular government department.

Data availability The data used in this study is not owned by the researchers and not publicly available. The data are de-identified linked government administrative data subject to data custodian and ethics approvals for access, linkage and use. Approval to access the same data can be sought through an application process administered by the independent data linkage authority, SA NT Datalink.

Funding Funding for this project was from the National Health and Medical Research Council of Australia's Partnership Project Grant (APP1056888) and Centre for Research Excellence (APP1099422).

Authors' relationships and activities The authors declare that there are no relationships or activities that might bias, or be perceived to bias, their work.

Contribution statement MB prepared the initial draft, reviewed and revised the manuscript, analysed the data, and helped in conceptualising and designing the study. LGS, RMP, CRC and JWL acquired the data, provided intellectual input in statistical analyses and interpretation of the findings, and critically reviewed and edited the draft. MP provided intellectual input, helped in interpreting the findings, and critically reviewed and edited the draft. All authors approved the final manuscript as submitted and agree to be accountable for all aspects of the work. Particularly MB, LGS, RMP and CRC accept full responsibility for the work, had access to the data and controlled the decision to publish.

\section{References}

1. Krischer JP, Lynch KF, Schatz DA et al (2015) The 6 year incidence of diabetes-associated autoantibodies in genetically at-risk children: the TEDDY study. Diabetologia 58(5):980-987. https://doi.org/10. 1007/s00125-015-3514-y

2. Stene LC, Gale EAM (2013) The prenatal environment and type 1 diabetes. Diabetologia 56:1888-1897

3. Atkinson MA, Eisenbarth GS, Michels AW (2014) Type 1 diabetes. Lancet 383:69-82

4. DIAMOND Project Group (2006) Incidence and trends of childhood type 1 diabetes worldwide 1990-1999. Diabet Med 23:857866

5. Redondo MJ, Yu L, Hawa M et al (2001) Heterogeneity of type I diabetes: analysis of monozygotic twins in Great Britain and the United States. Diabetologia 44(3):354-362. https://doi.org/10. 1007/s001250051626

6. Joubert BR, Felix JF, Yousefi P et al (2016) DNA methylation in newborns and maternal smoking in pregnancy: Genome-wide consortium meta-analysis. Am J Hum Genet 98(4):680-696. https://doi.org/10.1016/j.ajhg.2016.02.019

7. Qiu F, Liang CL, Liu H et al (2017) Impacts of cigarette smoking on immune responsiveness: up and down or upside down? Oncotarget 8(1):268-284. https://doi.org/10.18632/oncotarget.13613

8. Patterson C, Guariguata L, Dahlquist G, Soltesz G, Ogle G, Silink M (2014) Diabetes in the young - a global view and worldwide estimates of numbers of children with type 1 diabetes. Diabetes Res Clin Pract 103(2):161-175. https://doi.org/10.1016/j.diabres. 2013.11.005

9. Reitan T, Callinan S (2017) Changes in smoking rates among pregnant women and the general female population in Australia, 
Finland, Norway, and Sweden. Nicotine Tob Res 19(3):282-289. https://doi.org/10.1093/ntr/ntw188

10. Australian Institute of Health and Welfare (2019) Trends in incidence of type 1 diabetes by age, 2000-2016. Data tables: Diabetes 2019. Available from www.aihw.gov.au/reports/diabetes/diabetessnapshot/data. Accessed 1 May 2019

11. Australian Institute of Health and Welfare, Laws PJ, Hilder L (2008) Australia's mothers and babies 2006. Perinatal statistics series no. 22. Cat. no. PER 46. Available from www.aihw.gov.au/ reports/mothers-babies/australias-mothers-babies-2006/contents/ table-of-contents. Accessed 1 May 2019

12. Australian Institute of Health and Welfare (2018) Australia's mothers and babies 2016 - in brief. Perinatal Statistics Series no. 34. Catalogue no. PER 97. Canberra: Australian Institute of Health and Welfare, 2018. Available from www.aihw.gov.au/reports/ mothers-babies/australias-mothers-babies-2016-in-brief/contents/ table-of-contents. Accessed 3 May 2019

13. Mattsson K, Jonsson I, Malmqvist E, Larsson HE, Rylander L (2015) Maternal smoking during pregnancy and offspring type 1 diabetes mellitus risk: accounting for HLA haplotype. Eur $\mathbf{J}$ Epidemiol 30(3):231-238. https://doi.org/10.1007/s10654-0149985-1

14. Robertson L, Harrild K (2010) Maternal and neonatal risk factors for childhood type 1 diabetes: a matched case-control study. BMC Public Health 10:281

15. Haynes A, Cooper MN, Bower C, Jones TW, Davis EA (2014) Maternal smoking during pregnancy and the risk of childhood type 1 diabetes in Western Australia. Diabetologia 57(3):469-472. https://doi.org/10.1007/s00125-013-3122-7

16. Magnus MC, Tapia G, Olsen SF et al (2018) Parental smoking and risk of childhood-onset type 1 diabetes. Epidemiology 29(6):848856. https://doi.org/10.1097/EDE.0000000000000911

17. Ievins R, Roberts SE, Goldacre MJ (2007) Perinatal factors associated with subsequent diabetes mellitus in the child: record linkage study. Diabet Med 24:664-670

18. Frederiksen B, Kroehl M, Lamb MM et al (2013) Infant exposures and development of type 1 diabetes mellitus: The Diabetes Autoimmunity Study in the Young (DAISY). JAMA Pediatr 167(9):808-815. https://doi.org/10.1001/jamapediatrics.2013.317

19. Adlercreutz EH, Wingren CJ, Vincente RP, Merlo J, Agardh D (2015) Perinatal risk factors increase the risk of being affected by both type 1 diabetes and coeliac disease. Acta Paediatr 104(2):178184. https://doi.org/10.1111/apa.12836

20. D'Angeli MA, Merzon E, Valbuena LF, Tirschwell D, Paris CA, Mueller BA (2010) Environmental factors associated with childhood-onset type 1 diabetes mellitus: an exploration of the hygiene and overload hypotheses. Arch Pediatr Adolesc Med 164(8):732-738. https://doi.org/10.1001/archpediatrics.2010.115

21. Svensson J, Carstensen B, Mortensen HB, Borch-Johnsen K (2005) Early childhood risk factors associated with type 1 diabetes-is gender important? Eur J Epidemiol 20(5):429-434. https://doi. org/10.1007/s10654-005-0878-1

22. Marshall AL, Chetwynd A, Morris JA et al (2004) Type 1 diabetes mellitus in childhood: a matched case control study in Lancashire and Cumbria, UK. Diabet Med 21(9):1035-1040. https://doi.org/ 10.1111/j.1464-5491.2004.01282.x

23. Nuske T, Pilkington R, Gialamas A, Chittleborough C, Smithers L, Lynch J (2016) The SA Early Childhood Data Project. Research Series. School of Public Health, The University of Adelaide, 2016. Available from https://health.adelaide.edu.au/betterstart/ publications/reports/sa-ecdp-report-2016.pdf Accessed 4 May 2019

24. Centre for Health Record Linkage (2012) Quality assurance. Available from www.cherel.org.au/quality-assurance. Accessed 7 February 2018

25. Australian Consortium for Classification Development (2017) The International Statistical Classification of Diseases and Related
Health Problems, tenth revision, Australian modification (ICD-10$\mathrm{AM} / \mathrm{ACHI} / \mathrm{ACS}$ ), 10th edn. Available from www.ihpa.gov.au/ what-we-do/icd-10-am-achi-acs-previous-editions. Accessed 2 May 2017

26. Scheil W, Jolly K, Scott J, Catcheside B, Sage L, Kennare R (2017) Pregnancy outcome in South Australia 2015. Available from www. sahealth.sa.gov.au/wps/wcm/connect/public+content/sa+health+ internet/about+us/health+statistics/pregnancy+outcome+statistics. Accessed 2 April 2018

27. McLean A (2001) Validation of the 1994 South Australian perinatal data collection form. Pregnancy Outcome Unit, Epidemiology Branch, Department of Human Services, Adelaide, SA

28. Lipsitch M, Tchetgen Tchetgen E, Cohen T (2010) Negative controls: a tool for detecting confounding and bias in observational studies. Epidemiology 21(3):383-388. https://doi.org/10.1097/ EDE.0b013e3181d61eeb

29. VanderWeele TJ, Ding P (2017) Sensitivity analysis in observational research: Introducing the E-value. Ann Intern Med 167(4):268 274. https://doi.org/10.7326/M16-2607

30. Sterne JA, White IR, Carlin JB et al (2009) Multiple imputation for missing data in epidemiological and clinical research: potential and pitfalls. BMJ 338:b2393

31. White IR, Royston P (2009) Imputing missing covariate values for the Cox model. Stat Med 28:1982-1998

32. Rubin DB (1987) Multiple imputation for nonresponse in surveys. Wiley, Hoboken

33. Persson S, Dahlquist G, Gerdtham UG, Steen Carlsson K (2013) Impact of childhood-onset type 1 diabetes on schooling: a population-based register study. Diabetologia 56(6):1254-1262. https://doi.org/10.1007/s00125-013-2870-8

34. Couper JJ, Haller MJ, Greenbaum CJ et al (2018) ISPAD Clinical Practice Consensus Guidelines 2018: stages of type 1 diabetes in children and adolescents. Pediatr Diabetes 19: 20-27

35. Banderali G, Martelli A, Landi M et al (2015) Short and long term health effects of parental tobacco smoking during pregnancy and lactation: a descriptive review. J Transl Med 13:327

36. Kiechl-Kohlendorfer U, Ralser E, Pupp Peglow U, Reiter G, Griesmaier E, Trawoger R (2010) Smoking in pregnancy: a risk factor for adverse neurodevelopmental outcome in preterm infants? Acta Paediatr 99(7):1016-1019. https://doi.org/10.1111/j.16512227.2010.01749.x

37. Australian Institute of Health and Welfare (2019) National Core Maternity Indicators. Smoking during pregnancy. Available from www.aihw.gov.au/reports/mothers-babies/ncmi-datavisualisations/contents/antenatal-period-indicators/smokingduring-pregnancy. Accessed 16 September 2019

38. Ekblad M, Gissler M, Korkeila J, Lehtonen L (2014) Trends and risk groups for smoking during pregnancy in Finland and other Nordic countries. Eur J Pub Health 24(4):544-551. https://doi.org/ 10.1093/eurpub/ckt128

39. Hidayat K, Zou S-Y, Shi B-M (2019) The influence of maternal body mass index, maternal diabetes mellitus, and maternal smoking during pregnancy on the risk of childhood-onset type 1 diabetes mellitus in the offspring: Systematic review and meta-analysis of observational studies. Obes Rev 20(8):1106-1120. https://doi.org/ 10.1111/obr.12858

40. Ram R, Morahan G (2017) Effects of type 1 diabetes risk alleles on immune cell gene expression. Genes 8:167

41. Penno MA, Couper JJ, Craig ME et al (2013) Environmental determinants of islet autoimmunity (ENDIA): a pregnancy to early life cohort study in children at-risk of type 1 diabetes. BMC Pediatr 13: 124

42. The Lancet, Diabetes \& Endocrinology (2019) Type 1 diabetes research: poised for progress. Lancet Diabetes Endocrinol 7:1-1 
43. Paul DS, Teschendorff AE, Dang MAN et al (2016) Increased DNA methylation variability in type 1 diabetes across three immune effector cell types. Nat Commun 7:13555

44. England L, Zhang J (2007) Smoking and risk of preeclampsia: a systematic review. Front Biosci 12:2471-2483. https://doi.org/10. $2741 / 2248$

45. Li X, Li W, Liu G, Shen X, Tang Y (2015) Association between cigarette smoking and Parkinson's disease: a meta-analysis. Arch Gerontol Geriatr 61(3):510-516. https://doi.org/10.1016/j.archger. 2015.08.004

46. Berkowitz L, Schultz BM, Salazar GA et al (2018) Impact of cigarette smoking on the gastrointestinal tract inflammation: Opposing effects in crohn's disease and ulcerative colitis. Front Immunol 9: 74-74

47. Ungprasert P, Crowson CS, Matteson EL (2016) Smoking, obesity and risk of sarcoidosis: A population-based nested case-control study. Respir Med 120:87-90. https://doi.org/10.1016/j.rmed. 2016.10.003
48. Xia Y, Kellems RE (2009) Is preeclampsia an autoimmune disease? Clin Immunol 133(1):1-12. https://doi.org/10.1016/j.clim.2009.05. 004

49. Cleynen I, Boucher G, Jostins L et al (2016) Inherited determinants of Crohn's disease and ulcerative colitis phenotypes: a genetic association study. Lancet 387(10014):156-167. https://doi.org/10.1016/ S0140-6736(15)00465-1

50. Haynes A, Bower C, Bulsara MK, Jones TW, Davis EA (2004) Continued increase in the incidence of childhood type 1 diabetes in a population-based Australian sample (1985-2002). Diabetologia 47(5):866-870. https://doi.org/10.1007/s00125-004-1385-8

51. Mattsson K, Kallen K, Rignell-Hydbom A et al (2016) Cotinine validation of self-reported smoking during pregnancy in the Swedish Medical Birth Register. Nicotine Tob Res 18(1):79-83. https://doi.org/10.1093/ntr/ntv087

Publisher's note Springer Nature remains neutral with regard to jurisdictional claims in published maps and institutional affiliations. 